\title{
Fault Diagnosis of Planetary Gear Based on Fuzzy Entropy of CEEMDAN and MLP Neural Network by Using Vibration Signal
}

\author{
Xi-Hui CHEN, Gang CHENG ${ }^{a}$, Chang LIU and Yong LI \\ School of Mechatronic Engineering, China University of Mining and Technology, Xuzhou, 221116, China
}

\begin{abstract}
A method of planetary gear fault diagnosis based on the fuzzy entropy of complete ensemble empirical mode decomposition with adaptive noise (CEEMDAN) and multi-layer perceptron (MLP) neural network is proposed. The vibration signal is decomposed into multiple intrinsic mode functions (IMFs) by CEEMDAN, and the fuzzy entropy that combines the fuzzy function and sample entropy is proposed and used to extract the feature information contained in each IMF. The fuzzy entropies of each IMF are defined as the input of the MLP neural network, and the planetary gear status can be recognized by the output. The experiments prove the proposed method is effective.
\end{abstract}

\section{Introduction}

Planetary gear often used in the key parts of the transmission system of large-scale complex equipment. However, it often suffered from the influences of heavy-duty and hostile environment, leading faults to occur [1]. Therefore, it is of great significance to study the fault diagnosis of planetary gears. Due to the influence of its complex structure, installation errors and operating environment, the vibration signal of planetary gear shows the characteristics with nonlinear and nonstationary [2]. Therefore, a fault feature extraction method that is suitable for processing the nonstationary vibration signal should be developed.

The time-frequency analysis method not only reflects the frequency characteristics of the signal but also shows the time-varying characteristics, making it is more suitable for the analysis of nonstationary vibration signals. Empirical mode decomposition (EMD) is a typical adaptive timefrequency analysis method, but it has a major drawback of modal aliasing [3]. With the development of research, ensemble empirical mode decomposition (EEMD) is proposed to solve the problem of modal aliasing. However, the reconstructed signal after EEMD contains more residual noise, and it is not a complete decomposition process. CEEMDAN is an improved EEMD algorithm, and the limited adaptive white noise is added at each decomposition stage. Each IMF is obtained by calculating the only remaining part, and the reconstruction error is almost zero. Therefore, CEEMDAN can overcome the modal aliasing phenomenon of EEMD, mitigating the incompleteness of EEMD, and a set of high quality and effective intrinsic mode function (IMF) can be obtained $[4,5]$.

Sample entropy is now applied in the fault feature quantification to reflect the complexity of the signal from the perspective of their similarity. However, in the calculation process of sample entropy,

\footnotetext{
${ }^{a}$ Corresponding author: chenxh@cumt.edu.cn
} 
the similarity of the signal is reflected to a dichotomy, exhibiting both similarity and dissimilarity, it is inaccurate to describe the similarity. So the fuzzy entropy is proposed [6]. The fuzzy function is used to describe the signal similarity, and a more accurate calculation for the signal similarity is achieved. The complexity and stability of the signal are described more reasonably and effectively. The recognition of planetary gear status is important after extracting the feature information, and some intelligent classification technologies are widely used [7]. The MLP neural network has great practical value in pattern recognition in many fields, and it is composed of an input layer, an output layer and many hidden layers. MLP neural network is a type of belong to multi-layer feed-forward neural network, and it realizes the data transformation of input features by the transfer functions of the hidden layers. MLP neural network can be applied to recognize different planetary gear statuses, generating a structured network to achieve highly complex nonlinear mapping by data self-learning [8]. Therefore, in this paper, a fault diagnosis method of planetary gear based on fuzzy entropy of CEEMDAN and MLP neural network is established.

\section{Model analysis}

\subsection{Complete ensemble empirical mode decomposition with adaptive noise}

EMD is originally proposed by Huang [3], but it has the drawback of modal aliasing. EEMD is proposed to solve this problem, and the Gaussian white noise is added into the original signal to change the distribution of extreme points. However, the reconstructed signal of the decomposition result of EEMD still contains more residual noise, and EEMD is not a complete decomposition process. Therefore, CEEMDAN is proposed, and the limited adaptive white noise is added at each decomposition stage. Each IMF is obtained by calculating the only remaining part, and the reconstruction error is zero. Therefore, CEEMDAN not only can overcome the modal aliasing, but can also eliminate the incompleteness of EEMD. The reconstructed signal is identical to the original signal $[9,10]$. The decomposition process of CEEMDAN can be described as follows:

Step1: Assume $E_{j}(\cdot)$ is defined as the first $j$ IMF that is obtained by EMD [3], and $\omega_{m}(t)$ is the white noise with a zero mean unit variance. For the signal $x(t)+\varepsilon_{0} \omega_{m}(t)$, the first IMF that is expressed as $\operatorname{IMF} 1(t)$ is obtained by EMD with $M$ experiments, and it is expressed as follows:

$$
\operatorname{IMF}_{1}(t)=\frac{1}{M} \sum_{m=1}^{M} E\left\{x(t)+\varepsilon_{0} \omega_{m}(t)\right\}
$$

where $\varepsilon_{0}$ is the amplitude coefficient of the white noise.

Step2: The first remaining part can be described as follows:

$$
r_{1}(t)=x(t)-\operatorname{IMF}_{1}(t)
$$

Step3: The computation process of the second IMF of the original signal $x(t)$ by CEEMDAN is expressed as follows:

$$
\operatorname{IMF}_{2}(t)=\frac{1}{M} \sum_{m=1}^{M} E_{1}\left\{r_{1}(t)+\varepsilon_{1} E_{1}\left(\omega_{m}(t)\right)\right\}
$$

Step4: For the other stages, namely, $k=2,3, \ldots, K$, the calculation process of the first $k$ remaining part is same as Step2 and Step3. The first $k+1$ IMF is as follows: 


$$
\left\{\begin{array}{l}
r_{k}(t)=r_{k-1}(t)-\operatorname{IMF}_{k}(t) \\
\operatorname{IMF}_{k+1}(t)=\frac{1}{M} \sum_{m=1}^{M} E_{1}\left\{r_{k}(t)+\varepsilon_{k} E_{k}\left(\omega_{m}(t)\right)\right\}
\end{array}\right.
$$

Step5: Step4 is continued until the remaining part of the signal can no longer be decomposed, and the criterion is that the number of extreme points is not more than two. The number of final IMFs is $K$, and the final remaining part is as follows:

$$
R(t)=x(t)-\sum_{k=1}^{K} \operatorname{IMF}_{k}(t)
$$

It can be seen from the realization process of CEEMDAN that the decomposition process is complete and can realize the reconstruction of the original signal. In each decomposition stage, the white noise can obtain an appropriate signal-to-noise ratio by adjusting the coefficient $\varepsilon_{k}$.

\subsection{Fuzzy entropy}

The calculation process of fuzzy entropy is as follows[11]:

Step1: Assuming $\operatorname{IMF}_{k}(t)=\{z(1), z(2), \ldots, z(n)\}$, a set of vectors that are used to calculate the fuzzy entropy is built, and they are expressed as follows:

$$
A_{i}=\{z(i), z(i+1), \ldots, z(i+m-1)\}-u_{0}(i) \quad i=1,2, \ldots, n-m+1
$$

where $m$ is the length of the vector. $u_{0}(i)$ is the mean of each vector.

Step2: The distances of each vector are calculated, and the distance between $A_{i}$ and $A_{j}$ can be defined as follows:

$$
d_{i j}^{m}\left(A_{i}, A_{j}\right)=\max \left(\left|A_{i}(l)-A_{j}(l)\right|\right) \quad l=1,2, \ldots, n
$$

Step3: The similarity between each set of vectors is described using a fuzzy function. An exponential function [12] is used, and it is defined as follows:

$$
D_{i j}^{m}=\mathrm{e}^{-\left(d_{i j}^{m} / r\right)^{n}}
$$

where $n$ is the boundary gradient of the exponential function, and $r$ is the similar tolerance.

Step4: The representation function $B^{m}$ is defined as follows:

$$
B^{m}=\frac{1}{n-m} \sum_{i=1}^{n-m}\left|\frac{1}{n-m-1} \sum_{j=1, j \neq i}^{n-m} D_{i j}^{m}\right|
$$

Step5: Make $m=m+1$ and repeat Step1-Step4, $B^{m+1}$ can be obtained, and the fuzzy entropy can be expressed as follows:

$$
F \mathrm{uzzy} E n=\ln ^{\left(B^{m} / B^{m+1}\right)}
$$

\subsection{Multi-Layer Perceptron Neural Network}

The MLP neural network is composed of input layer, output layer and hidden layer, and the training process of the MLP neural network $[13,14]$ is expressed as follows: 
Step1: The connection weights of the MLP neural network are initialized. The inputted features are sent to the hidden layer, and the calculation of each neuron of the hidden layer is expressed as follows:

$$
s_{h}=f\left(\sum_{j}^{J} x i_{j} W_{j h}+\theta_{h}\right)
$$

where $x i_{j}$ is the $j$-th input feature, $W_{j h}$ is the connection weight between input neuron $j$ and hidden neuron $h, \theta_{h}$ is the bias of the $h$-th hidden neuron. $f()$ is the activation function.

Step2: The calculation result of the hidden neuron is transmitted to the output layer, and the calculation of each neuron of the output layer is expressed as follows:

$$
y o_{k}=g\left(\sum_{h}^{H} s_{h} W_{h k}+\eta_{k}\right)
$$

where $s_{h}$ is the output of the $h$-th hidden neuron, $W_{h k}$ is the connection weight between hidden neuron $h$ and output neuron $k, \eta_{k}$ is the bias of the $k$-th output neuron.

Step3: Each output neuron has a target pattern corresponding to an input pattern $t_{k}$, and the error information of the output neuron is calculated as $\delta_{k}=t_{k}-y o_{k}$. The error information of the hidden layer is then calculated as:

$$
\delta_{h}=\left(\sum_{k=1}^{K} \delta_{k} W_{h k}\right) s_{h}
$$

Step4: The weight updating of the hidden neurons and the output neurons is expressed as follows:

$$
\begin{aligned}
& W_{j h}(t+1)=W_{j h}(t)+\alpha \delta_{h} x i_{j}+\mu\left[W_{j h}(t)-W_{j h}(t-1)\right] \\
& W_{h k}(t+1)=W_{h k}(t)+\alpha \delta_{k} s_{h}+\mu\left[W_{h k}(t)-W_{h k}(t-1)\right]
\end{aligned}
$$

where $\alpha$ is the learning rate, and $\mu$ is a momentum factor.

Step5: Determine whether the system meets the stop condition.

\section{Test equipment and data acquisition}

The fault simulation experiment of the planetary gear is carried out, and the basic layout of the experiment is shown in Figure 1. In the experiment, the motor speed is set to $40 \mathrm{~Hz}$, and the load is set to $13.5 \mathrm{Nm}$. Five types of sun gears are simulated, and they are normal gear, broken gear, gear with one missing tooth, wear gear and gear with a tooth root crack. The sampling frequency is set to 12800 $\mathrm{Hz}$, and the vibration signals of the five types of gears are collected. 


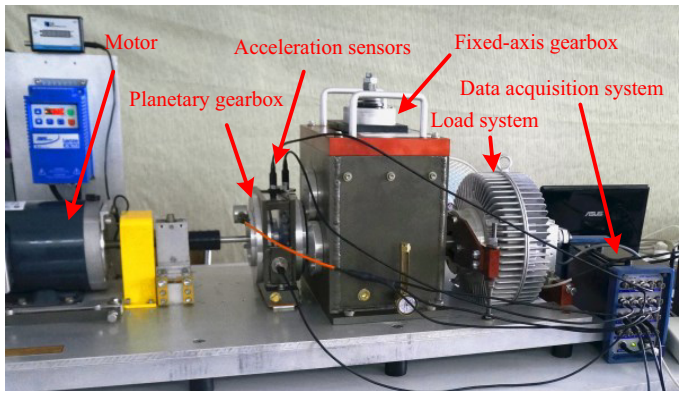

Figure 1. Fault experiment for the planetary gear

\section{Experiment signal analys is}

The five types of vibration signals for different gears are collected and shown in Figure 2.

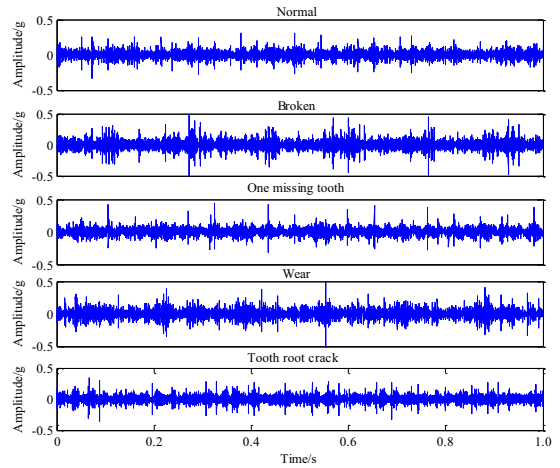

Figure 2. Five types of vibration signals for different gears

It can be seen from Figure 2 that five types of vibration signals for different gears have no obvious difference. It is not able to distinguish the gear status by comparing the time domain vibration signals, next, they are processed by the proposed method to verify the effectiveness of the method. The vibration signals are decomposed by CEEMDAN, and due to the limitation of space, the vibration signal of the broken gear is selected as an example to show the CEEMDAN process. To prove that the performance of CEEMDAN is superior to that of EEMD, the decomposition results using EEMD and CEEMDAN for broken gear are shown in Figure 3. It can be seen from Figure 3 that the vibration signal is decomposed into 12 IMFs and a residual signal, and for convenience of expression, the residual signal is called IMF13. IMF1-IMF13 are arranged from high frequency to low frequency. The decomposition result of EEMD still exhibits a serious modal aliasing phenomenon, as shown in IMF6, IMF7, IMF8 and IMF9. Meanwhile, some of them even are the illusive components, such as in IMF7 and IMF8. In the decomposition result using CEEMDAN, the quality of the obtained IMFs is greatly improved, and the modal aliasing phenomenon is further suppressed. 


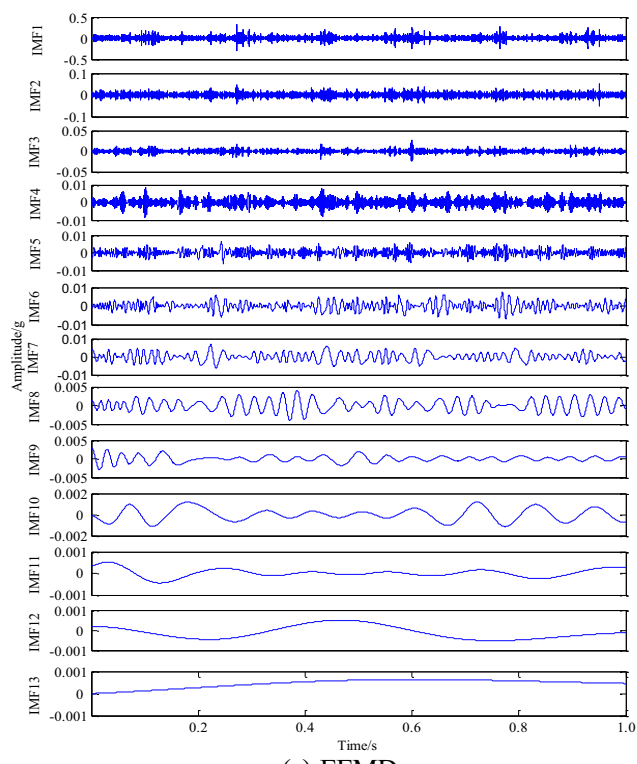

(a) EEMD

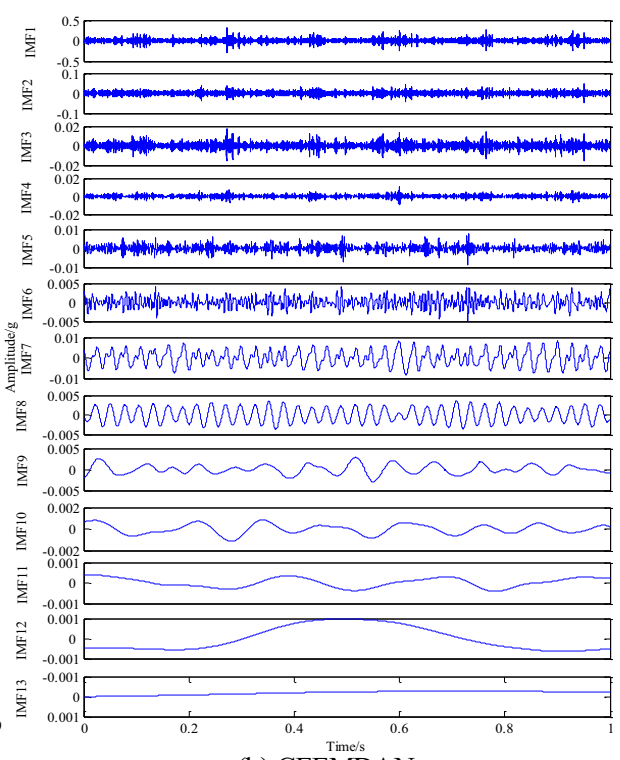

(b) CEEMDAN

Figure 3. Decomposition result using EEMD and CEEMDAN for broken gear

Fuzzy entropy combining a fuzzy function and sample entropy is used to quantize the feature information contained in IMFs, and the fuzzy function is used to describe the similarity of each vector. The exponential function $D_{i j}^{m}=\mathrm{e}^{-\left(d_{i j}^{m} / r\right)^{n}}$ is selected, in which there are some parameters that need to be determined: the similar tolerance $r$, boundary gradient $n$ and length of the compared vectors $m . r$ is usually set by the standard deviation of the signal, and in this paper, $r=0.15 \mathrm{~s} d . n$ is usually recommended to take a smaller integer value, so in this paper, $n$ is set as $2 . m$ is determined as 7000 after some experiments. To prove that fuzzy entropy has a better performance than sample entropy, the sample entropy and fuzzy entropy of each IMF are shown in Figure 4, respectively.

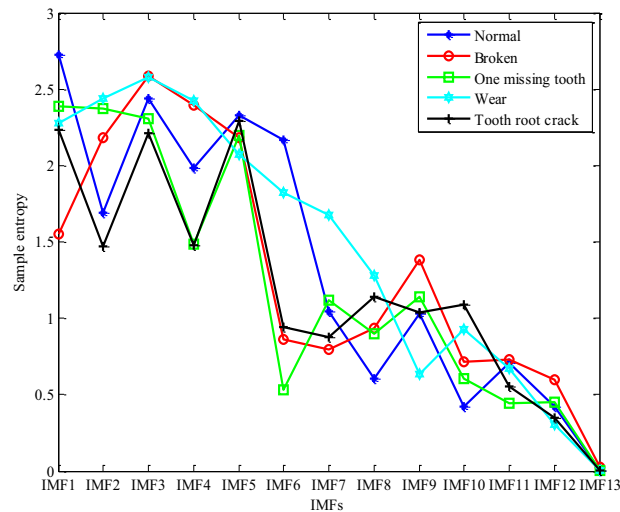

(a) Sample entropy

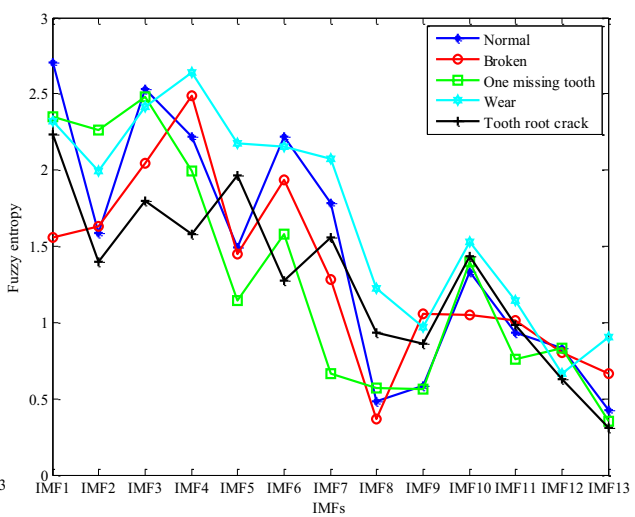

(b) Fuzzy entropy

Figure 4. Sample entropy and fuzzy entropy of each IMF 
It can be seen from Figure 4(a) that the complexity and stability of each IMF are described by the sample entropy. The sample entropies of the five types of gears are scattered in some IMFs, for example, IMF2 and IMF10, but in most of the IMFs, the sample entropies of some gears overlap together, for example, IMF3 and IMF4, etc. Especially in IMF5, the sample entropies of the five types of gears even coincide at a point, and those situations will interfere with the identification of five types of gears. In the calculation process of the sample entropy, the similarity description of the compared vectors is dichotomous, that causes the similarity of the compared vectors can not be described accurately, and the tiny differences among the signals can not be reflected effectively. The Fuzzy entropy is proposed, and the fuzzy function is used to calculate the similarity of the compared vectors in the calculation process. It can be seen from Figure 4(b) that the fuzzy entropies of the five types of gears are scattered in most of the IMFs. Only in IMF11 and IMF12, the overlap phenomenon is appear, but the overlap phenomenon is relatively minor. It can be found that the fuzzy entropy more easily distinguishes between the five types of gears, and it is thus superior to the sample entropy in the aspect of feature extraction.

Recognizing the planetary gear status is the most important step after extracting the feature information. Next, the fault diagnosis of the planetary gear is accurately achieved by applying a MLP neural network. The training sample set is prepared, and each planetary gear status has 30 samples, for a total of 150 samples. The fuzzy entropies of each IMF decomposed by CEEMDAN are defined as the input of MLP neural network, so that the input layer of MLP neural network has 13 input neurons. Because the training samples are divided into five types, so the output layer of MLP neural network has 5 output neurons. To train MLP neural network, different planetary gear statuses are denoted with different labels in the output layer: the normal gear is denoted as $\left[\begin{array}{lllll}1 & 0 & 0 & 0 & 0\end{array}\right]$, the broken gear is denoted as $\left[\begin{array}{lllll}0 & 1 & 0 & 0 & 0\end{array}\right]$, the gear with one missing tooth is denoted as $\left[\begin{array}{lllll}0 & 0 & 1 & 0 & 0\end{array}\right]$, the wear gear is denoted as $\left[\begin{array}{lllll}0 & 0 & 0 & 1 & 0\end{array}\right]$ and the gear with tooth root crack is denoted as $\left[\begin{array}{lllll}0 & 0 & 0 & 0 & 1\end{array}\right]$. The neuron number of the hidden layer is usually determined by trial-and-error. The learning rate of MLP neural network is set as 0.5 , and the training step is set as 80 . The mean squared error is used to evaluate the training performance of MLP neural network when the training process is completed. The changes in the mean squared error of the training process with different numbers of hidden neurons are shown in Figure 5(a). A testing sample set that includes different planetary gear status samples is prepared, and each planetary gear status has 40 samples, for a total of 200 samples. They are decomposed by CEEMDAN, and the fuzzy entropies of each IMF are inputted into the trained MLP neural network for use in verifying the recognition performance of the trained MLP neural network. The overall recognition rate for planetary gear with different numbers of hidden neurons is shown in Figure 5(b).

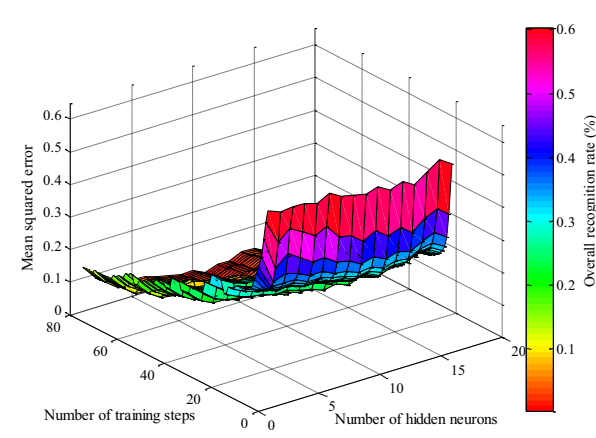

(a) Mean squared error

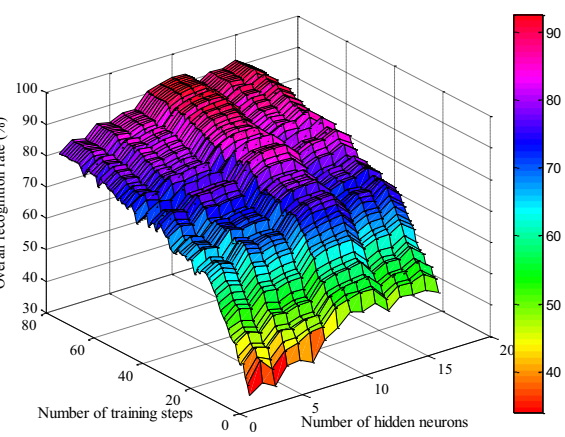

(b) Overall recognition rate

Figure 5. Mean squared error and overall recognition rate with different numbers of hidden neurons

It can be seen from Figure 5(a) that the mean squared error declines with the increase of the training steps, and it tends to stabilize when the training step is between 65 and 80 , indicating that the training process of MLP neural network is completed. The final mean squared error has a minimum 
value when the MLP neural network has 11 hidden neurons, and it is 0.0035 . It can be seen from Figure 5(b) that the overall recognition rate with different numbers of hidden neurons increases with the number of training steps, but the final overall recognition rate has a difference for different numbers of hidden neurons. The overall recognition rate for the planetary gear has a maximum value when the MLP neural network has 11 hidden neurons, and it reaches $91 \%$. Hence, the MLP neural network with 11 hidden neurons has the best performance for the fault diagnosis of the planetary gear, and the detailed recognition rates of the MLP neural network with 11 hidden neurons for the different planetary gears are shown in Table 1.

Table 1. Detailed recognition rates of the MLP neural network with 11 hidden neurons

\begin{tabular}{cccccc}
\hline & Normal gear & Broken gear & $\begin{array}{c}\text { Gear with } \\
\text { one missing tooth }\end{array}$ & Wear gear & $\begin{array}{c}\text { Gear with } \\
\text { tooth root crack }\end{array}$ \\
\hline Recognition rate & $92.5 \%$ & $95 \%$ & $90 \%$ & $87.5 \%$ & $90 \%$ \\
\cline { 2 - 6 } Overall recognition rate & \multicolumn{5}{c}{$91 \%$} \\
\hline
\end{tabular}

It can be seen from Table 1 that the overall recognition rate of the MLP neural network with 11 hidden neurons reaches $91 \%$. Of different planetary gear statuses, the broken gear has the highest recognition rate, and it reaches $95 \%$. While the wear gear has the lowest recognition rate, and it reaches $87.5 \%$. The recognition rates of the normal gear, gear with one missing tooth and gear with tooth root crack are $92.5 \%, 90 \%$ and $90 \%$, respectively. The experiments prove the planetary gear faults can be recognized by the proposed method based on the fuzzy entropy of CEEMDAN and MLP neural network, making it an effective fault diagnosis method for planetary gears.

\section{Conclusions}

A method of planetary gear fault diagnosis based on the fuzzy entropy of CEEMDAN and MLP neural network is proposed. CEEMDAN is used to decompose the vibration signal into multiple IMFs, the quality of the obtained IMFs and the completeness of the decomposition process are greatly improved. Fuzzy entropy combining a fuzzy function and sample entropy is used to quantize the feature information contained in the IMFs, and it can be found from data analysis that the fuzzy sample has a better performance than that of sample entropy, making it more suitable for extracting the feature information. The fuzzy entropies of each IMF are defined as the input of MLP neural network, and the mean squared error is selected as a criterion to train MLP neural network. The testing sample set is used to verify the recognition performance of the trained MLP neural network, and the overall recognition rate with different numbers of hidden neurons is calculated. The overall recognition rate for planetary gear has a maximum value when the MLP neural network has 11 hidden neurons, and it reaches $91 \%$. The recognition rate of the broken gear reaches $95 \%$, and followed by normal gear, gear with one missing tooth, gear with tooth root crack and wear gear. These results show that the proposed fault diagnosis method based on the fuzzy entropy of CEEMDAN and MLP neural network has a better recognition performance for planetary gear, making it an effective fault diagnosis method for planetary gears.

\section{Acknowledgment}

This work was supported by a Project Funded by the Priority Academic Program Development of Jiangsu Higher Education Institutions, the Natural Science Foundation of Jiangsu Province (grant number BK20141128) and Fundamental Research Funds for the Central Universities (grant number 2014ZDPY31); this support is gratefully acknowledged. 


\section{References}

1. Y.G. Lei, D.T. Kong, J. Lin, M.J. Zuo, Fault detection of planetary gearboxes using new diagnostic parameters, Meas. Sci. Technol., 23 (2012) 055605-055615.

2. K.E. Ko, D.H. Lim, P.Y. Kim, J. Park, A study on the bending stress of the hollow sun gear in a planetary gear train, J. Mech. Sci. Technol., 24 (2010) 29-32.

3. N.E. Huang, Z. Shen, S.R. Long, The empirical mode decomposition and the Hilbert spectrum for nonlinear and non-stationary time series analysis, Proc. R. Soc. London., 454 (1998) 903-995.

4. X.M. Xue, J.Z. Zhou, Y.H. Xu, W.L. Zhu, C.S. Li, An adaptively fast ensemble empirical mode decomposition method and its applications to rolling element bearing fault diagnosis, Mech. Syst. Signal Proc., 62-63 (2015) 444-459.

5. J.R. Yeh, J.S. Shieh, N.E. Huang, Complementary ensemble empirical mode decomposition: a novel noise enhanced data analysis method, Adv. Adapt. Data Anal., 2(2) (2010) 135-156.

6. L. Zhang, G. Xiong, H. Liu, Bearing fault diagnosis using multi-scale entropy and adaptive neuro-fuzzy inference, Expert Syst. Appl., 37 (2010) 6077-6085.

7. W. Guo, L.J. Huang, C. Chen, H.W. Zou, Z.W. Liu, Elimination of end effects in local mean decomposition using spectral coherence and applications for rotating machinery, Digit. Signal Prog., 55 (2016) 52-63.

8. M. Akhoondzadeh, A MLP neural network as an investigator of TEC time series to detect seismoionospheric anomalies, Adv. Space. Res., 51 (2013) 2048-2057.

9. X.H. Chen, G. Cheng, X.L. Shan, X. Hu, Q. Guo, H.G. Liu, Research of weak fault feature information extraction of planetary gear based on ensemble empirical mode decomposition and adaptive stochastic resonance, Measurement, 73 (2015) 55-67.

10. M.A. Colominasa, G. Schlotthauera, M.E. Torresa, Improved complete ensemble EMD: A suitable tool for biomedical signal processing. Biomed. Signal Process. Control, 14 (2014) 19-29.

11. D.R. Kong, H.B. Xie, Use of modified sample entropy measurement to classify ventricular tachycardia and fibrillation, Measurement, 44(4) (2011) 653-662.

12. J.D. Zheng, J.S. Cheng, Y. Yang, S.R. Luo, A rolling bearing fault diagnosis method based on multi-scale fuzzy entropy and variable predictive model-based class discrimination, Mech. Mach. Theory, 78(16) (2014) 187-200.

13. S. Souahlia, K. Bacha, A. Chaari, MLP neural network-based decision for power transformers fault diagnosis using an improved combination of rogers and doernenburg ratios DGA, Electr. Power Energy Syst., 43 (2012) 1346-1353.

14. V.N. Ghate, S.V. Dudul, Optimal MLP neural network classifier for fault detection of three phase induction motor, Expert Syst. Appl., 37 (2010) 3468-3481. 